\title{
Systemic use of tumor necrosis factor alpha as an anticancer agent
}

\author{
Nicholas J. Roberts ${ }^{1}$, Shibin Zhou ${ }^{1}$, Luis A. Diaz Jr. ${ }^{1}$ and Matthias Holdhoff ${ }^{1}$ \\ ${ }^{1}$ Ludwig Center for Cancer Genetics and Therapeutics, The Sidney Kimmel Comprehensive Cancer Center, The Johns \\ Hopkins University, Baltimore, MD 21231, USA \\ Correspondence to: Matthias Holdhoff, email: mholdhol@jhmi.edu
}

Keywords: cancer, chemotherapy, TNF, drug combinations, oncotarget

Received: October 17, 2011, Accepted: October 26, 2011, Published: October 27, 2011

Copyright: @ Roberts et al. This is an open-access article distributed under the terms of the Creative Commons Attribution License, which permits unrestricted use, distribution, and reproduction in any medium, provided the original author and source are credited.

ABSTRACT:

Tumor necrosis factor-a (TNF-a) has been discussed as a potential anticancer agent for many years, however initial enthusiasm about its clinical use as a systemic agent was curbed due to significant toxicities and lack of efficacy. Combination of TNF-a with chemotherapy in the setting of hyperthermic isolated limb perfusion (ILP) has provided new insights into a potential therapeutic role of this agent. The therapeutic benefit from TNF-a in ILP is thought to be not only due to its direct anti-proliferative effect, but also due to its ability to increase penetration of the chemotherapeutic agents into the tumor tissue. New concepts for the use of TNF-a as a facilitator rather than as a direct actor are currently being explored with the goal to exploit the ability of this agent to increase drug delivery and to simultaneously reduce systemic toxicity.

This review article provides a comprehensive overview on the published previous experience with systemic TNF-a. Data from 18 phase I and 10 phase II single agent as well as 18 combination therapy studies illustrate previously used treatment and dose schedules, response data as well as the most prominently observed adverse effects. Also discussed, based on recent preclinical data, is a potential future role of systemic TNF-a in combination with liposomal chemotherapy to facilitate increased drug uptake into tumors.

\section{INTRODUCTION}

TNF- $\alpha$ was discovered in 1975 and subsequently cloned in 1984 [1,2] and has been the focus of considerable interest as an anticancer agent. Initial enthusiasm for TNF- $\alpha$ as a systemic therapeutic agent stemmed from the observation that it could induce hemorrhagic necrosis in the tumors of bacillus Calmette-Guérin (BCG)-primed and endotoxin treated mice [1]. Recombinant human TNF- $\alpha($ rhTNF- $\alpha)$ has been tested as a systemic treatment in several phase I and phase II clinical trials. These trials, conducted in the 1980s and 1990s, used TNF- $\alpha$ as both a single agent and in combination with other cytokines or chemotherapeutics. However, the initial enthusiasm for the development of TNF- $\alpha$ as a systemic treatment has waned in the face of significant toxicities and a lack of evidence for therapeutic benefit. Nevertheless, these studies have provided valuable data regarding the toxicity profile and pharmacological properties of systemically delivered TNF- $\alpha$. More encouraging is the current clinical use of combination TNF- $\alpha$ with chemotherapeutic agents in the setting of hyperthermic isolated limb perfusion in limb-threatening soft tissue sarcomas and in-transit melanoma [3]. Here we review the phase I and phase II clinical trials of systemic use of TNF- $\alpha$, the toxicities and responses observed, and highlight recent scientific advances that hint at reduced systemic toxicities and augmentation of the antitumor responses seen with this agent. Specifically, the recently identified vascular effects of TNF- $\alpha$ that lead to a targeted intra-tumoral enrichment of liposomes and macromolecules through an enhancedenhanced permeability and retention effect (E $\left.{ }^{2} \mathrm{PR}\right)$ [4-6].

\section{BIOLOGY OF TNF- $\alpha$}

TNF- $\alpha$ is a 23 kilodalton (kDa) type II transmembrane protein arranged in stable homotrimers. A $51 \mathrm{kDa}$ soluble homotrimeric cytokine is derived from 
the transmembrane form via proteolytic cleavage by the metalloprotease TNF- $\alpha$ converting enzyme (TACE) [7]. TNF- $\alpha$ is primarily produced by macrophages, but also by a variety of other cells, including NK cells, T lymphocytes, smooth muscle cells, fibroblasts and others [8]. Release of TNF- $\alpha$ occurs in response to inflammatory stimuli and cytokines including peptidoglycan, lipopolysaccharide and other bacterial components [9]. Two receptors exist for TNF- $\alpha$ : 1). Tumor necrosis factor receptor 1 (TNFR1), which preferentially binds soluble TNF- $\alpha$ and is found almost ubiquitously on the surface of cells, and 2). Tumor necrosis factor receptor 2 (TNFR2), which is found on cells of the hematopoietic lineage and which has specificity for the transmembrane form of TNF- $\alpha$ [10]. The resulting biological effect of TNF- $\alpha$ binding to its receptor is depending on the type of receptor activated and the cellular state during activation. Stimulation of TNFR1 activates downstream inflammatory mediators through AP1, MAPK and NF-kB pathways [11]. The balance of activation of these pathways by TNF- $\alpha$ is critical in determining whether a cell undergoes apoptosis as a late stage event in TNF- $\alpha$ stimulation. For instance, in acute myeloid leukemia (AML), NF-kB dependent induction of HO-1 underlies resistance to TNF- $\alpha$ induced apoptosis [12]. Similarly, inhibition of NF-kB with concurrent TNF- $\alpha$ stimulation results in caspase activation and apoptosis [13]. Conversely, the biological role and downstream effects of TNFR2 stimulation are more poorly understood. TNFR2 can be up-regulated by cytokine stimulation and also mediate a variety of downstream inflammatory mediators [14].

\section{PRECLINICAL EVIDENCE FOR TNF- $\alpha$ AS AN ANTICANCER AGENT}

After the initial observation that TNF- $\alpha$ induced hemorrhagic necrosis of tumors in mice treated with BCG and endotoxin [1], the potential of TNF- $\alpha$ as a therapeutic agent was intensely studied in in vitro and in vivo studies. These studies highlighted the possible role of TNF- $\alpha$ as an anticancer agent and galvanized support for the numerous phase I and phase II studies that followed.

In vitro studies demonstrated that $\mathrm{TNF}-\alpha$ had a growth inhibitory effect on SV40-tranformed human mammary epithelia cells and a cytotoxic effect on breast cancer cell lines. Interestingly, there was no effect on normal human mammary epithelial cells [15]. Similarly, TNF- $\alpha$ showed a cytostatic effect on hepatoma cells while having little effect on non-tumorigenic liver cells [16]. Intriguingly, Sugarman and colleagues showed that the cytostatic and cytotoxic effects of TNF- $\alpha$ were cell line specific, with only a proportion of tumor cell lines responding to TNF- $\alpha$ [17]. Comparison of the cytostatic and cytotoxic effect of TNF- $\alpha$ against a wide range of tumor types demonstrated that approximately a quarter of tumors $(28 \%)$ are sensitive to the effects of TNF- $\alpha$ and that this sensitivity was greater in colorectal and lung cancers [18]. In vivo, TNF- $\alpha$ has shown activity against a wide variety of murine tumor types and human tumor xenografts [19-21]. Taken together, the in vitro and in vivo data were suggestive that TNF- $\alpha$ had the potential to be highly specific anti-cancer therapy, with activity against a number of tumor types.

Preclinical evidence also suggested a synergistic effect of TNF- $\alpha$ with a variety of chemotherapeutics in vitro and in vivo. TNF- $\alpha$ has been shown to enhance the cytotoxicity of DNA topoisomerase inhibitors actinomycin D, adriamycin, and etoposide against murine bladder tumor cell line (MBT-2) in in vitro and in vivo models [22]. The enhancing effect of TNF- $\alpha$ was not observed with other cytotoxic agents, such as: bleomycin, hydroxyurea, cisplatin, mitomycin $\mathrm{C}$, vincristine and vinblastine. The timing of TNF- $\alpha$ treatment in relation to chemotherapy seems important with studies suggesting that the optimal time for TNF- $\alpha$ therapy is 48 hours prior to initiation of chemotherapy [23]. Interestingly, there are likely two mechanisms that underlie the importance of timing in regards to TNF- $\alpha$ treatment. Firstly, inhibitors of transcription, such as actinomycin D and flavopiridol, are used before or at the time of TNF- $\alpha$ treatment and block NF-kB pathway activation, sensitizing cells to the effects of TNF- $\alpha$ [24]. Secondly, inhibitors of topoisomerase II can be given at the time of, or after TNF- $\alpha$ and increase the sensitivity of TNF- $\alpha$ resistant cancer cell lines to TNF- $\alpha[25]$.

TNF- $\alpha$ also demonstrated enhanced antitumor effects in vitro when used in combination with other cytokines [26]. Induction of TNF- $\alpha$ receptors rather than an increased affinity of already present receptors explained the effect of IFN- $\gamma$ on TNF- $\alpha$ binding $[26,27]$. Similarly, in vitro and in vivo studies using the TNF- $\alpha$ resistant melanoma cell line B16BL6 demonstrated that IFN- $\gamma$ sensitizes cancer cells to the effects of TNF- $\alpha$, inducing necrosis and tumor response, which were previously absent [28]. Later, investigators showed that TNF- $\alpha$ induced synergistic growth inhibition against pancreatic cancer cell lines when combined with interferon alpha (IFN- $\alpha$ ) and IFN- $\gamma$ [29]. TNF- $\alpha$ and IFN- $\gamma$ act against many other cancer cell lines as well. Orita et al. [30] tested TNF- $\alpha$ and IFN- $\gamma$ on 23 cell lines in vitro and demonstrated that the combination acts synergistically, showing cytostatic and cytotoxic effects on cell lines previously resistant to TNF- $\alpha$ and IFN- $\gamma$ individually.

Combinations of TNF- $\alpha$ with IFN- $\alpha$ and IL-2 also showed synergistic cytotoxic and cytostatic effects in vitro and in vivo. Concomitant TNF- $\alpha$ and IFN- $\alpha$ in a murine lung metastasis model significantly increased survival [31]. TNF- $\alpha$ and IL-2 in murine models with leukemia, mastocytoma, melanoma, lymphoma and sarcoma cell lines also demonstrate combinatorial effects and systemic immunological memory $[32,33]$.

The combination of TNF- $\alpha$ and radiotherapy has been 
Table 1: Phase I studies with single agent rhTNF- $\alpha$

\begin{tabular}{|c|c|c|c|c|c|c|c|}
\hline Study & $\begin{array}{c}\text { Total } \\
\text { number of } \\
\text { patients }\end{array}$ & $\begin{array}{l}\text { Tumor } \\
\text { Type }\end{array}$ & Dose TNF- $a^{a}$ & Schedule & ORR' & MTD & $\begin{array}{l}\text { Dose Limiting } \\
\text { Toxcities }\end{array}$ \\
\hline Chapman 1987 [35] & 13 & $\begin{array}{l}\text { Advanced } \\
\text { cancer }\end{array}$ & $\begin{array}{c}1-200 \mu g / m^{2} \\
\text { for (IV bolus) } \\
\text { and } 5-250 \\
\mu g / m^{2}(S Q)\end{array}$ & $\begin{array}{l}\text { Twice weekly alternating } \\
\text { SQ/IV rhTNF- } \alpha \text { ev ery week for } \\
4 \text { weeks }\end{array}$ & $8 \%$ & NR & $\begin{array}{l}\text { Hy potension. Local } \\
\text { tissue reaction. Nausea. } \\
\text { Vomiting. Neurotoxicity. }\end{array}$ \\
\hline Creaven 1987 [36] & 29 & $\begin{array}{l}\text { Adv anced } \\
\text { cancer - } \\
\text { solid tumors }\end{array}$ & $\begin{array}{l}1 \times 10^{4}-48 \times \\
10^{4} \text { units } / \mathrm{m}^{2}\end{array}$ & Three doses 3 weeks apart & $0 \%$ & $\begin{array}{l}48 \times 10^{4} \\
\text { units } / \mathrm{m}^{2}\end{array}$ & Hy potension. \\
\hline Kim ura 1987 [37] & 33 & $\begin{array}{l}\text { Adv anced } \\
\text { cancer - } \\
\text { solid tumors }\end{array}$ & $\begin{array}{l}1 \times 10^{5}-16 \\
\times 10^{5} \text { units } / \mathrm{m}^{2}\end{array}$ & One dose & $0 \%$ & $\begin{array}{l}5 \times 10^{5} \\
\text { units } / \mathrm{m}^{2}\end{array}$ & $\begin{array}{l}\text { Hy potension. } \\
\text { Thrombocy topenia. } \\
\text { Hepatotoxicity. }\end{array}$ \\
\hline Creagan 1988 [38] & 27 & $\begin{array}{l}\text { Adv anced } \\
\text { cancer - } \\
\text { solid tumors }\end{array}$ & $\begin{array}{c}5-200 \\
\mu \mathrm{g} / \mathrm{m}^{2} / \mathrm{day}\end{array}$ & $\begin{array}{l}\text { Daily for } 5 \text { consecutive days } \\
\text { ev ery } 2-3 \text { weeks }\end{array}$ & $4 \%$ & $150 \mu \mathrm{g} / \mathrm{m}^{2}$ & $\begin{array}{l}\text { Hy potension. Rigors. } \\
\text { Phlebitis. }\end{array}$ \\
\hline Feinberg 1988 [39] & 39 & $\begin{array}{l}\text { Metastatic } \\
\text { cancer }\end{array}$ & $\begin{array}{c}5-250 \\
\mu \mathrm{g} / \mathrm{m}^{2} / \text { day }\end{array}$ & $\begin{array}{l}\text { Daily for five consecutive days } \\
\text { ev ery two weeks for } 8 \text { weeks. } \\
30 \text { minute vs. } 4 \text { hour inf usion }\end{array}$ & $0 \%$ & $200 \mu \mathrm{g} / \mathrm{m}^{2} /$ day & $\begin{array}{l}\text { Hy potension. Nausea. } \\
\text { Vomiting. My algias. } \\
\text { Fatigue. }\end{array}$ \\
\hline Sherman 1988 [40] & 19 & $\begin{array}{l}\text { Adv anced } \\
\text { cancer - } \\
\text { solid tumors }\end{array}$ & $\begin{array}{c}0.5 \times 10^{4}-3.0 \\
\times 10^{5} \\
\text { units } / \mathrm{m}^{2} / \text { day }\end{array}$ & $\begin{array}{l}\text { 5-day continuous infusion } \\
\text { every } 4 \text { weeks }\end{array}$ & $0 \%$ & $\begin{array}{r}3.0 \times 10^{5} \\
\text { units } / \mathrm{m}^{2} / \mathrm{d}\end{array}$ & $\begin{array}{l}\text { Thrombocy topenia. } \\
\text { Leukopenia. }\end{array}$ \\
\hline Spriggs 1988 [41] & 50 & $\begin{array}{l}\text { Advanced } \\
\text { cancer }\end{array}$ & $4.5-645 \mu \mathrm{g} / \mathrm{m}^{2}$ & $\begin{array}{l}\text { Continuous inf usion over } 24 \\
\text { hours ev ery } 3 \text { weeks }\end{array}$ & $2 \%$ & $636 \mu \mathrm{g} / \mathrm{m}^{2}$ & Hy potension. \\
\hline Taguchi 1988 [42] & $53^{c}$ & $\begin{array}{l}\text { Malignant } \\
\text { tumors }\end{array}$ & $\begin{array}{c}0.1 \times 10^{6}-5 \times \\
10^{6} \text { units/dose } \\
\text { (IV); } 0.1 \times 10^{6}- \\
2 \times \\
10^{6} \text { units/dose } \\
\text { (IT) }\end{array}$ & $\begin{array}{l}\text { One dose for week } 1 \text {, then } \\
\text { three times a week for week } \\
2-7\end{array}$ & $5 \%$ & $\begin{array}{c}1 \times 10^{6} \\
\text { units } / \text { dose }^{d}\end{array}$ & Hy potension. \\
\hline Creaven 1989 [43] & 33 & $\begin{array}{l}\text { Adv anced } \\
\text { cancer }\end{array}$ & $\begin{array}{r}5-80 \times 10^{4} \\
\text { units } / \mathrm{m}^{2} / \text { day }\end{array}$ & Daily for 5 consecutive days & $6 \%$ & $\begin{array}{c}60 \times 10^{4} \\
\text { units } / \mathrm{m}^{2} / \text { day }\end{array}$ & $\begin{array}{l}\text { Hy potension. } \\
\text { Hepatotoxicity. }\end{array}$ \\
\hline $\begin{array}{c}\text { Jakubowski } 1989 \\
\text { [44] }\end{array}$ & 19 & $\begin{array}{l}\text { Advanced } \\
\text { cancer }\end{array}$ & $\begin{array}{c}5-200 \\
\mu \mathrm{g} / \mathrm{m}^{2} / \mathrm{day}(\mathrm{IM})\end{array}$ & $\begin{array}{l}\text { Daily for } 5 \text { consecutive days } \\
\text { ev ery } 2 \text { weeks }\end{array}$ & $0 \%$ & $150 \mu \mathrm{g} / \mathrm{m}^{2} / \mathrm{day}$ & $\begin{array}{l}\text { Local injection site } \\
\text { reaction. Leukopenia. } \\
\text { Thrombocy topenia. } \\
\text { Hepatoxicity. } \\
\text { Neurotoxicity. }\end{array}$ \\
\hline $\begin{array}{c}\text { Wie denmann } 1989 \\
\text { [45] }\end{array}$ & 15 & $\begin{array}{l}\text { Adv anced } \\
\text { cancer - } \\
\text { adenocarcin } \\
\text { oma }\end{array}$ & $40-400 \mu \mathrm{g} / \mathrm{m}^{2}$ & $\begin{array}{l}\text { Continuous inf usion over } 24 \\
\text { hours once or twice weekly for } \\
8 \text { weeks }\end{array}$ & $0 \%$ & $200 \mu \mathrm{g} / \mathrm{m}^{2}$ & $\begin{array}{l}\text { Thrombocy topenia. } \\
\text { Fever. Cholls. Fatigue. } \\
\text { My algia. }\end{array}$ \\
\hline Gam m 1991 [46] & 62 & $\begin{array}{l}\text { Advanced } \\
\text { cancer }\end{array}$ & $2.5-200 \mu \mathrm{g} / \mathrm{m}^{2}$ & $\begin{array}{c}\text { Twice daily for } 5 \text { consecutive } \\
\text { days ev ery two weeks for } 8 \\
\text { weeks }\end{array}$ & $6 \%$ & $\begin{array}{c}150 \\
\mu \mathrm{g} / \mathrm{m}^{2} / \text { dose }\end{array}$ & $\begin{array}{l}\text { Hy potension. } \\
\text { Hepatotoxicity. }\end{array}$ \\
\hline Krige I 1991 [47] & 27 & $\begin{array}{l}\text { Adv anced } \\
\text { cancer - } \\
\text { solid tumors }\end{array}$ & $\begin{array}{l}8.5-1000 \\
\mu \mathrm{g} / \mathrm{m}^{2}\end{array}$ & $\begin{array}{c}100 \% \text { dose on day } 1, \text { then } \\
20 \% \text { of initial dose on day } 8 \text { - } \\
\text { day } 12 \text { repeated ev ery } 2 \\
\text { weeks }\end{array}$ & $0 \%$ & $\begin{array}{c}267 \mu \mathrm{g} / \mathrm{m}^{2} \\
\text { (initial dose) } \\
\text { and } 160 \mu \mathrm{g} / \mathrm{m}^{2} \\
\text { (subsequent } \\
\text { daily dosing) }\end{array}$ & $\begin{array}{l}\text { Hy potension. } \\
\text { Hemorrhagic gastritis. }\end{array}$ \\
\hline Logan 1991 [48] & 24 & $\begin{array}{l}\text { Adv anced } \\
\text { cancer - } \\
\text { solid tumors }\end{array}$ & $40-240 \mu \mathrm{g} / \mathrm{m}^{2}$ & $\begin{array}{l}100 \% \text { dose on day } 1 \text {, then } \\
\text { daily dosing on day } 8 \text { - day } 12 \\
\text { repeated ev ery } 3 \text { weeks }\end{array}$ & NR & NR & NR \\
\hline Schiller 1991 [49] & 53 & $\begin{array}{l}\text { Advanced } \\
\text { cancer }\end{array}$ & $5-275 \mu \mathrm{g} / \mathrm{m}^{2}$ & $\begin{array}{c}\text { Three times a week for } 4 \\
\text { weeks }\end{array}$ & $2 \%$ & $225 \mu \mathrm{g} / \mathrm{m}^{2}$ & $\begin{array}{l}\text { Hy potension. Fatigue. } \\
\text { Nausea. }\end{array}$ \\
\hline Mitte Iman 1992 [50] & 19 & $\begin{array}{l}\text { Adv anced } \\
\text { cancer - } \\
\text { solid tumors }\end{array}$ & $40-200 \mu \mathrm{g} / \mathrm{m}^{2}$ & $\begin{array}{c}24 \text {-hour infusion on day } 1 \\
\text { followed by } 120 \text {-hour inf usion } \\
\text { day } 8 \text { - day } 12 \text { repeated ev ery } \\
3 \text { weeks }\end{array}$ & $0 \%$ & $160 \mu \mathrm{g} / \mathrm{m}^{2}$ & $\begin{array}{l}\text { Hematologic toxicity. } \\
\text { Neurotoxicity. }\end{array}$ \\
\hline Furman 1993 [51] & 27 & $\begin{array}{l}\text { Pediatric } \\
\text { advanced } \\
\text { cancer }\end{array}$ & $\begin{array}{r}100-350 \\
\mu \mathrm{g} / \mathrm{m}^{2} / \mathrm{day}\end{array}$ & $\begin{array}{l}\text { Daily for } 5 \text { consecutive days } \\
\text { ev ery two weeks }\end{array}$ & $4 \%$ & $300 \mu \mathrm{g} / \mathrm{m}^{2} /$ day & $\begin{array}{l}\text { Cardiotoxicity. } \\
\text { Hy potension. } \\
\text { Hepatotoxicity. }\end{array}$ \\
\hline $\begin{array}{c}\text { Braczkowski } 1998 \\
\text { [52] }\end{array}$ & 21 & $\begin{array}{l}\text { Adv anced } \\
\text { cancer - } \\
\text { solid tumors }\end{array}$ & $75-150 \mu g /$ day & $\begin{array}{l}\text { Daily for } 5 \text { consecutive days } \\
\text { ev ery two weeks }\end{array}$ & $48 \%$ & N/A & NR \\
\hline
\end{tabular}


less extensively studied. Investigation of the interaction of TNF- $\alpha$ and radiation in 14 human tumor cells lines demonstrated synergistic or additive cytotoxicity with the maximum effect when TNF- $\alpha$ was given 4-12 hours before irradiation [34]. The mechanism of this synergism is thought to be due to the induction of oxygen free radical species and resulting DNA damage.

\section{CLINICAL TRIALS OF SYSTEMIC RECOMBINANT HUMAN TNF- $\alpha$}

\section{Systemic rhTNF- $\alpha$ as a single agent}

Numerous phase I and phase II studies have been conducted to ascertain the toxicity profile and efficacy of

Table 2: Side effects of single agent rhTNF-a

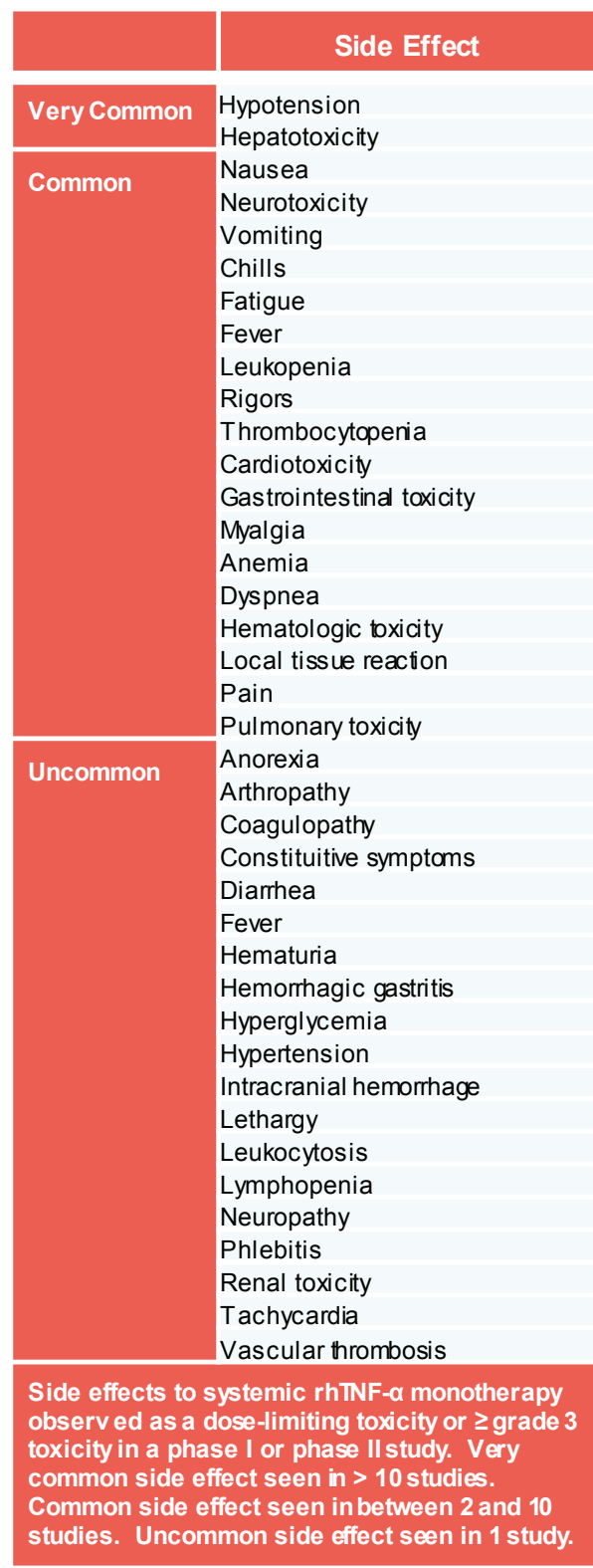

systemic TNF- $\alpha$. Studies have encompassed a wide range of tumor types in both adult and pediatric patients. In the majority of phase I and phase II studies, TNF- $\alpha$ was administered as an intravenous bolus injection or infusion. However, a few phase I studies have evaluated TNF- $\alpha$ with subcutaneous or intramuscular administration.

Phase I studies conducted with TNF- $\alpha$ are detailed in Table 1 [35-52]. Eighteen phase I studies were conducted and published with rhTNF- $\alpha$ as a single agent systemic therapy, enrolling between 19 and 62 patients per study. Study design varied with single dose of rhTNF- $\alpha$, multiple dosing (daily to every three weeks) and continuous infusion (one to five day duration) being tested. Overall, it appears that a systemic TNF- $\alpha$ dose of $150-200 \mu \mathrm{g} / \mathrm{m}^{2}$, given as a 30 minute intravenous infusion was identified as MTD in several studies. Dose-limiting toxicities (DLT) as well as other side effects that were observed seemed to have been universal and in most cases reasonably well tolerated and reversible. Common DLTs included: hypotension, thrombocytopenia, leukopenia, neurotoxicity, fever, nausea/vomiting, as well as general symptoms of malaise and weakness (Table 2). Other pathological sequelae of a transient hypovolemic episode, including transient elevation of liver enzymes, were reported. Tumor responses however, when used as a single agent, even with more intense treatment schedules, were rare.

Phase II studies using systemically administered rhTNF- $\alpha$ are detailed in Table 3 [53-62]. Studies typically investigated advanced/metastatic cases of: colorectal cancer, breast cancer, pancreatic cancer, malignant melanoma and renal cell carcinoma. The majority of studies involved a small number of cases (16-26), an exception being a phase II study of various malignancies that enrolled 147 patients [59]. Study design varied, with $150-200 \mu \mathrm{g} / \mathrm{m}^{2}$ given as a 30 minute intravenous infusion daily for 3-5 days and repeated every 1-4 weeks being commonly employed. In all studies, tumor responses were rare and when they did occur, only partial responses were observed. In the largest study of 147 cancer patients treated with $150 \mu \mathrm{g} / \mathrm{m}^{2}$ for 5 days every other week, only 1 partial remission was noted while $13 \%$ of patients experienced a grade 4 or greater toxicity. The most serious toxicities included respiratory failure and coagulopathies. Other, less serious and more common side effects reported include: hypotension (31\%), leukopenia (38\%), thrombocytopenia (13\%), fever / chills (69\%), headache (25\%), nausea / vomiting (69\%) and hepatopathy (10\%). However, compared to other phase II studies this regimen was fairly dose-dense which may have increased the significant toxicity observed.

\section{Systemic rhTNF-a in combination with chemotherapeutics}

Phase I and II studies that investigated the safety 
Table 3: Phase II studies with single agent rhTNF- $\alpha$

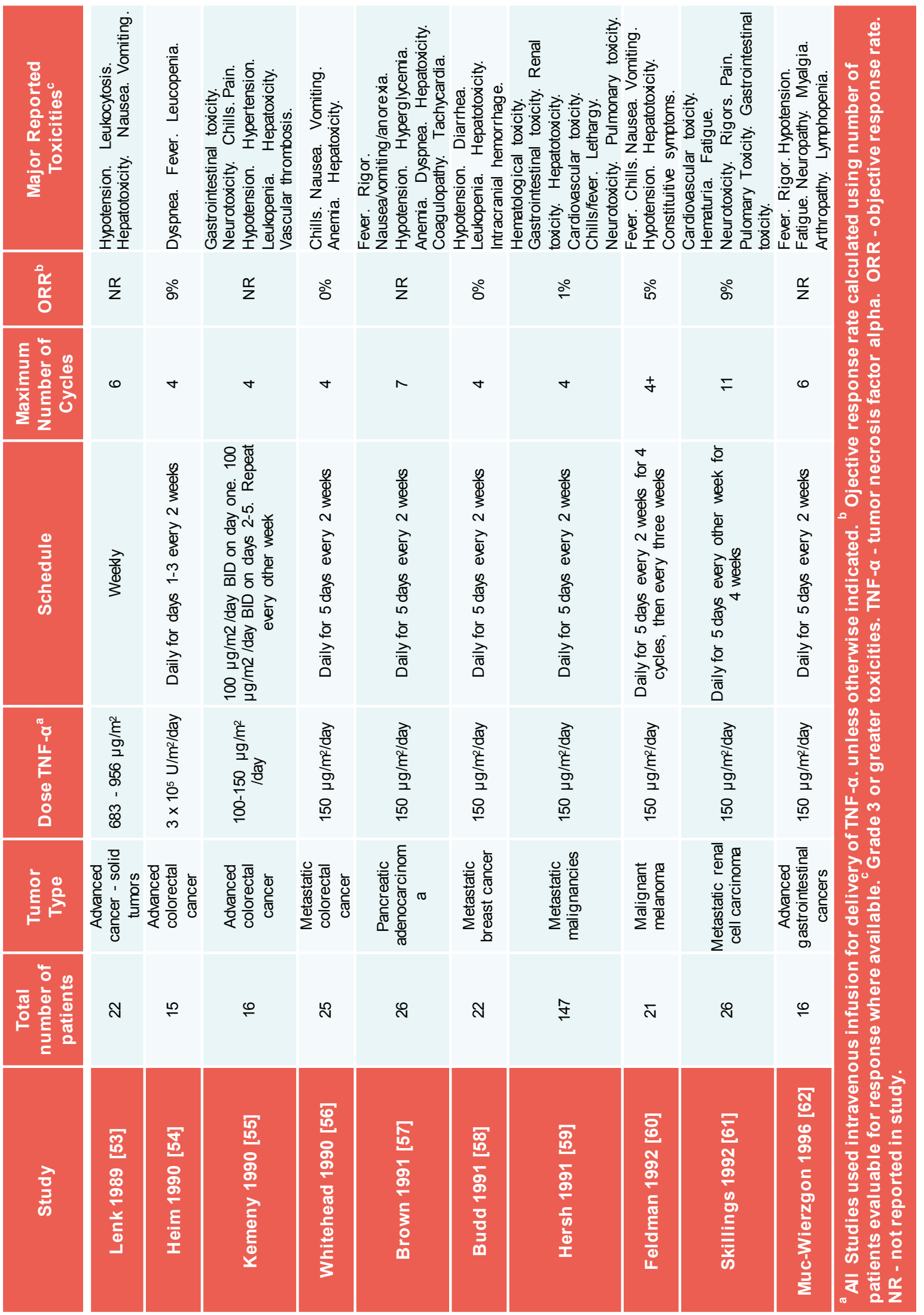


Table 4: Studies of systemic TNF- $\alpha$ with chemotherapy

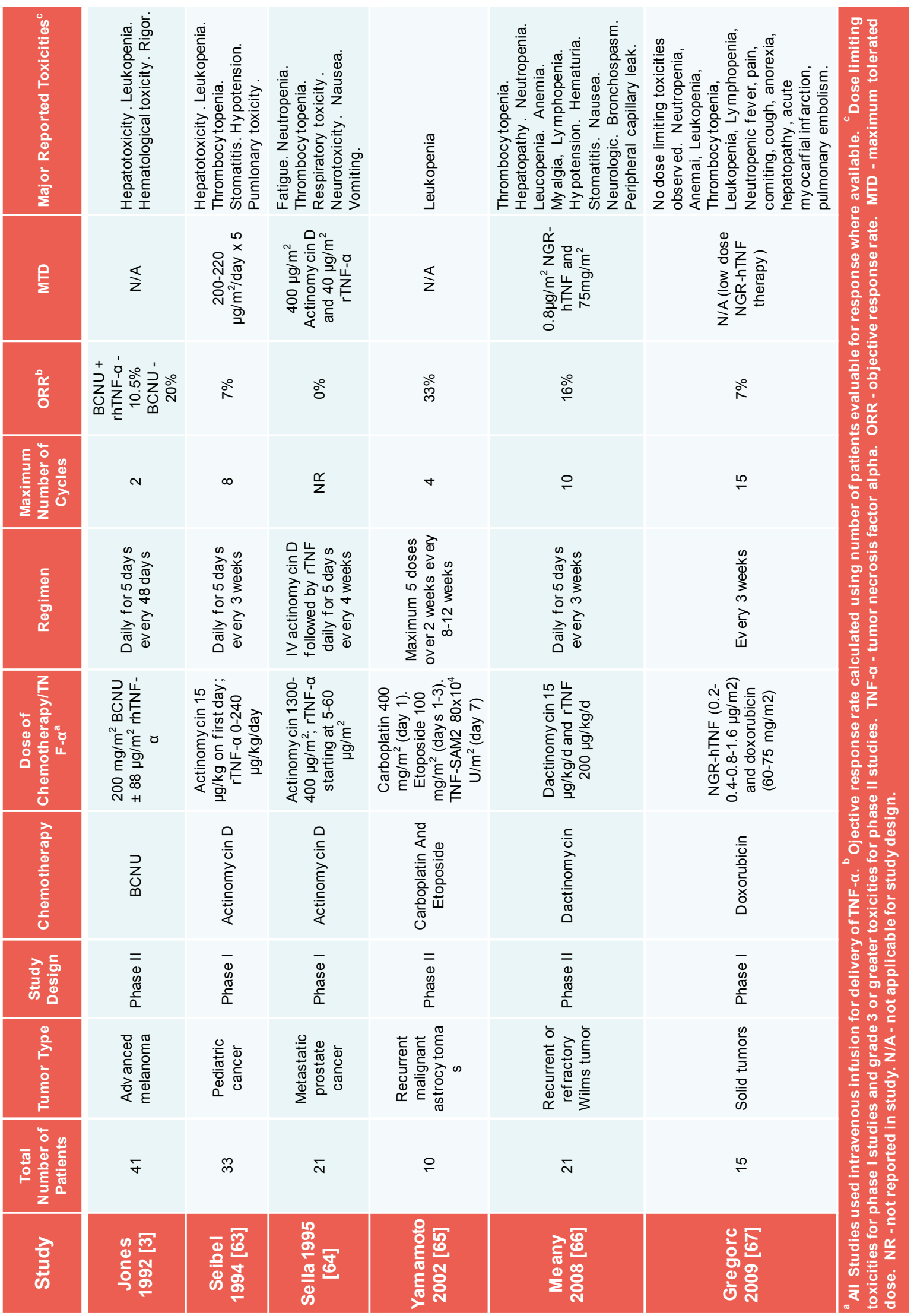


Table 5: Studies of systemic TNF- $\alpha$ with other cytokines

\begin{tabular}{|c|c|c|c|c|c|c|c|c|c|c|}
\hline 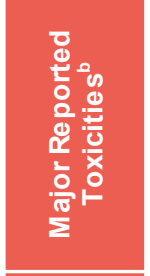 & 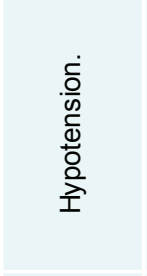 & 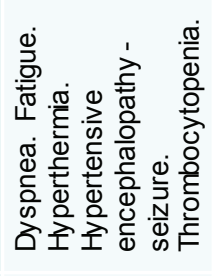 & 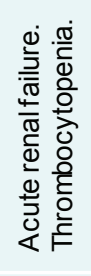 & 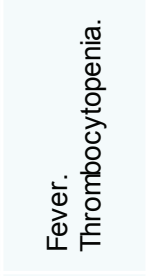 & 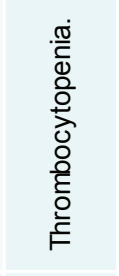 & 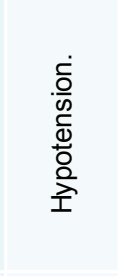 & 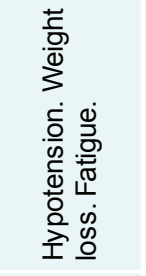 & 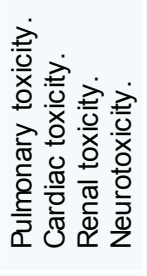 & 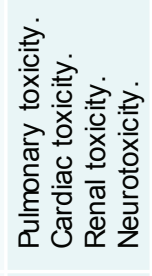 & 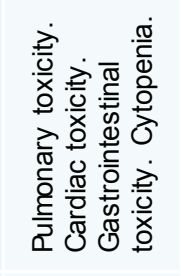 \\
\hline$\frac{\text { 을 }}{\mathbf{E}}$ & 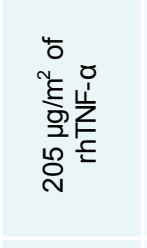 & 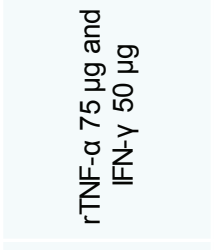 & 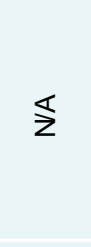 & 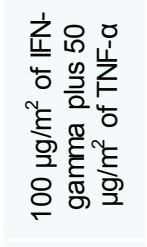 & 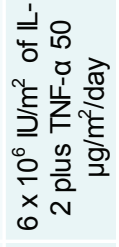 & 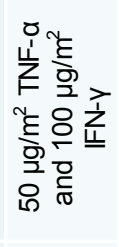 & 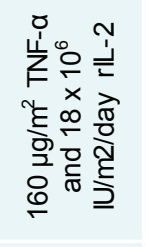 & $\$$ & 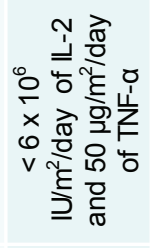 & 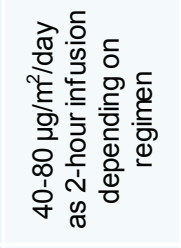 \\
\hline$\frac{\pi}{\alpha}$ & ๖े & ஃ̊ & ஃ̊ & $\frac{\alpha}{z}$ & ळ & $\frac{\alpha}{z}$ & $\stackrel{\stackrel{\circ}{\leftarrow}}{\leftarrow}$ & ১े & ○े & ஃ̊ \\
\hline $\begin{array}{l}\text { ब } \\
\frac{0}{0} \\
0 \\
0\end{array}$ & $\frac{\alpha}{z}$ & $N$ & $\nabla$ & 0 & $\sim$ & $\nabla$ & $\sim$ & $\sim$ & $\sigma$ & $\stackrel{\alpha}{z}$ \\
\hline $\begin{array}{l}\frac{5}{0} \\
\frac{5}{5} \\
\frac{9}{\alpha}\end{array}$ & 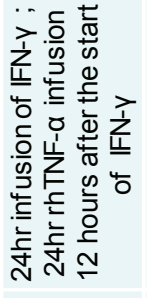 & 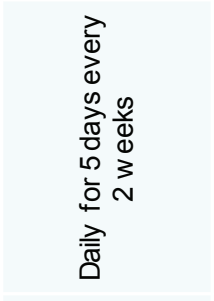 & 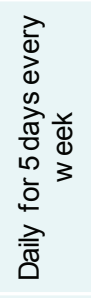 & 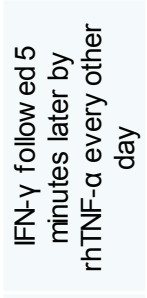 & 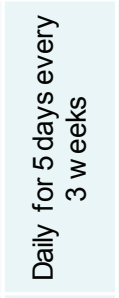 & 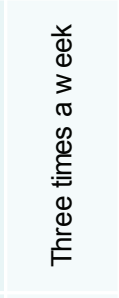 & 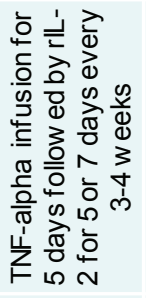 & 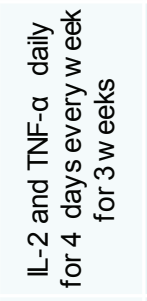 & 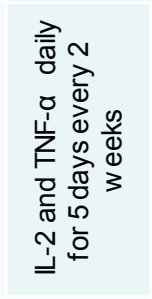 & 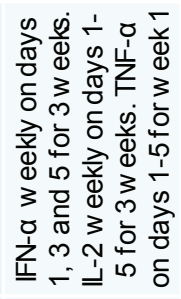 \\
\hline ํㅗㅇ 음 & 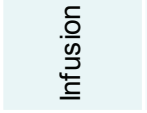 & 응 & 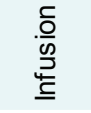 & $\frac{\mathscr{0}}{\mathrm{D}}$ & 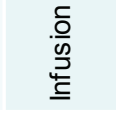 & 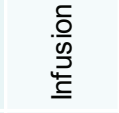 & 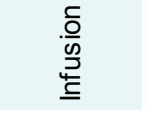 & $\frac{\mathscr{D}}{\overline{0}}$ & $\frac{0}{\mathrm{O}}$ & 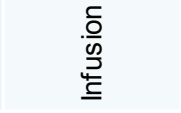 \\
\hline 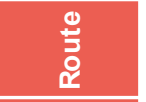 & $\geq$ & $\leqq$ & $\geq$ & $\geqq$ & $\geqq$ & $\geq$ & $\geq$ & $\geqq$ & $\geqq$ & $\geq$ \\
\hline 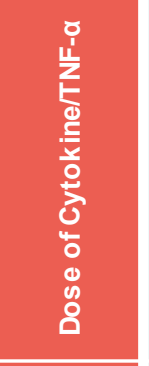 & 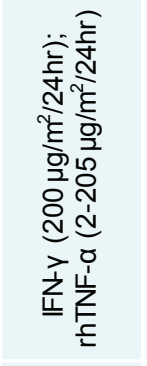 & 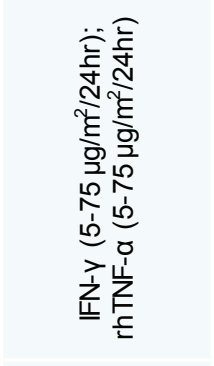 & 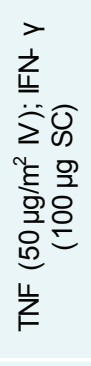 & 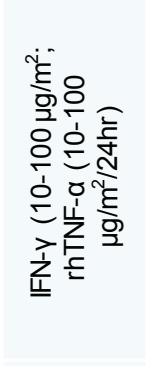 & 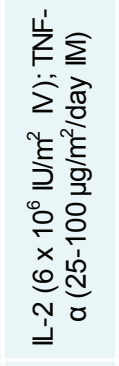 & 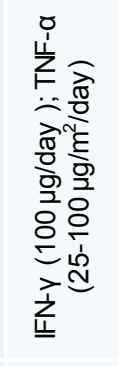 & 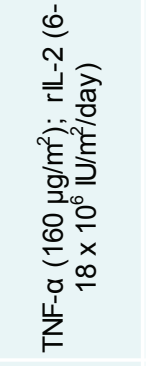 & 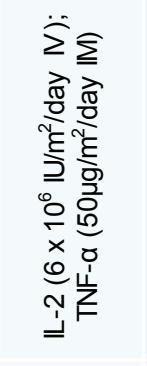 & 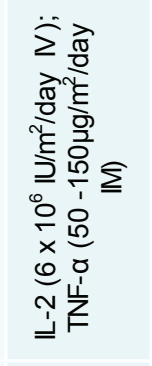 & 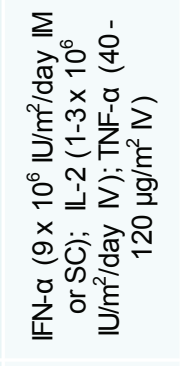 \\
\hline $\begin{array}{l}\frac{9}{2} \\
\frac{1}{0} \\
0 \\
0\end{array}$ & $\sum_{\underline{\underline{Z}}}^{\vec{z}}$ & $\sum_{\underline{\underline{Z}}}^{>}$ & $\sum_{\underline{\underline{Z}}}^{>}$ & $\sum_{\underline{\underline{Z}}}^{\overrightarrow{\rangle}}$ & $\stackrel{\sim}{\Perp}$ & $\sum_{\underline{1}}^{>}$ & $\stackrel{\sim}{\Perp}$ & $\stackrel{\sim}{\stackrel{N}{\Perp}}$ & $\stackrel{\sim}{\stackrel{N}{=}}$ & 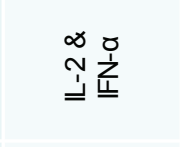 \\
\hline 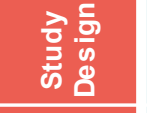 & $\begin{array}{l}\bar{D} \\
0 D \\
\mathbb{D} \\
\bar{L}\end{array}$ & $\begin{array}{l}\overline{0} \\
\infty \\
\mathbb{D} \\
\frac{D}{1}\end{array}$ & 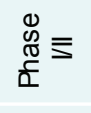 & $\begin{array}{l}\overline{1} \\
\mathbb{N} \\
\mathbb{D} \\
\bar{L}\end{array}$ & $\begin{array}{l}\bar{D} \\
\mathbb{D} \\
\mathbb{D} \\
\bar{L}\end{array}$ & $\begin{array}{l}\bar{\Phi} \\
\mathbb{D} \\
\bar{D} \\
\bar{D}\end{array}$ & $\begin{array}{l}\bar{D} \\
\mathbb{D} \\
\mathbb{D} \\
\bar{D}\end{array}$ & 흠 & 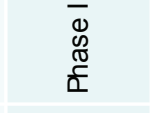 & $\begin{array}{l}\bar{D} \\
\mathbb{D} \\
\mathbb{D} \\
\bar{D}\end{array}$ \\
\hline 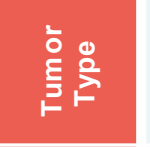 & 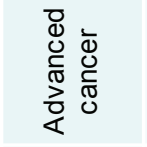 & 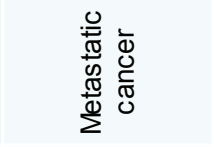 & 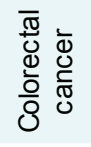 & 응 & 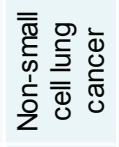 & 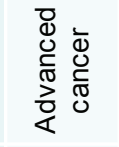 & 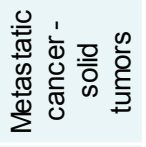 & 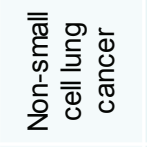 & 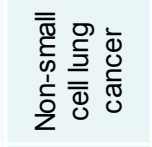 & 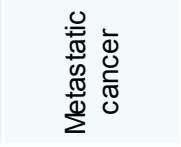 \\
\hline 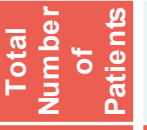 & ల్లొ & $\stackrel{\llcorner}{\sim}$ & $\div$ & m & $\stackrel{6}{\circ}$ & $\sim$ & - & $\infty$ & $\Lambda$ & $\stackrel{\infty}{-}$ \\
\hline$\frac{2}{\frac{2}{3}}$ & 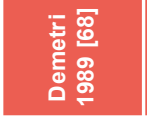 & 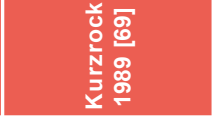 & 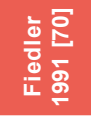 & 乒距 & 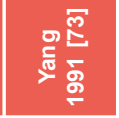 & 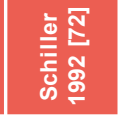 & 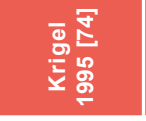 & 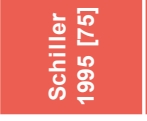 & 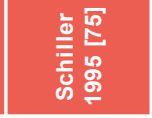 & 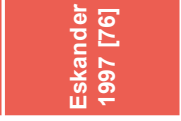 \\
\hline
\end{tabular}


and efficacy of systemic rhTNF- $\alpha$ combined with carmustine [3], actinomycin D [63, 64], carboplatin and etoposide [65], dactinomycin [66] and doxorubicin [67] have been reported and are detailed in Table 4 . In all trials, intravenous rhTNF- $\alpha$ was given concurrently or sequentially to chemotherapeutics on multiple days and treatments being repeated for a number of cycles. Dose of intravenous rhTNF- $\alpha$ ranged from $88-200 \mu \mathrm{g} / \mathrm{m}^{2}$ and was similar to the dose used for rhTNF- $\alpha$ monotherapy. In one study of rhTNF- $\alpha$ and BCNU in advanced melanoma, a response rate of $20 \%$ was seen with BCNU alone compared to $10.5 \%$ with BCNU and rhTNF- $\alpha$ [3]. Additionally, treatment of recurrent or refractory Wilms tumor with dactinomycin and rhTNF- $\alpha$ resulted in a $15.8 \%$ response rate [66]. However, while patients in this study were previously treated with dactinomycin, response to therapy was not conclusively due to the action of rhTNF- $\alpha$. Together, trials of rhTNF- $\alpha$ combined with chemotherapeutics have failed to prove that the addition of rhTNF- $\alpha$ to the treatment regimen improved outcome.

\section{Systemic rhTNF- $\alpha$ in combination with cytokines}

Many studies have combined systemic administration of rhTNF- $\alpha$ with other cytokines such as: IFN- $\gamma$ [68-72], IL-2 [73-76] and IFN- $\alpha$ [76], and these are summarized in Table 5. In general, phase I studies showed a reduction in the MTD of rhTNF- $\alpha$ when used in combination with other cytokines for patients with advanced solid tumors. This was largely due to the overlap in toxicities of these cytokines, that is: hypotension, fever, thrombocytopenia, acute renal failure, anemia, cardiac arrhythmias and pulmonary edema. Disappointingly, few objective responses were reported and none of the combinations were tested in larger randomized phase II studies; most likely because of the toxicity associated with combined therapy and a lack of efficacy seen in the initial studies.

\section{Systemic rhTNF-a in combination with radiotherapy}

Three studies that combined rhTNF- $\alpha$ with external beam radiation have also been reported [77-79] and they are detailed in Table 6. The most recent studies combined radiotherapy with both rhTNF- $\alpha$ and the chemotherapeutic ranimustine for the treatment of malignant astrocytoma [79]. In these studies DLTs were not observed and consequently, the maximum tolerated dose of this regimen was difficult to ascertain. In addition, no synergy in terms of objective response was noted.

\section{FUTURE DIRECTIONS OF SYSTEMIC TNF- $\alpha$}

Translation of systemic TNF- $\alpha$ from research to clinic has been hampered by significant systemic toxicity and a lack of efficacy at MTD $[43,46]$. Future directions for the development of TNF- $\alpha$ therapy rely on amelioration of the toxicity seen with systemic therapy and thereby increasing direct tumor response through higher TNF- $\alpha$ doses. Alternatively, the exploitation of novel mechanisms of action may increase efficacy and safety through indirect tumor effects.

Polyethylene glycol (PEG) conjugated proteins have shown increased retention and decreased immunogenicity in vivo [80]. Attempts to conjugate rhTNF- $\alpha$ with PEG has yielded a therapeutic with decreased toxicity and increased efficacy in murine preclinical models [8184]. Thamm and colleagues conducted a phase I clinical trial of PEG-rhTNF- $\alpha$ in dogs with spontaneously occurring tumors [85]. Comparatively, Client-owned dogs provide an excellent model in which to develop novel anticancer agents. These dogs are genetically diverse, immunocompetent, share our environment and have similar types and size of tumors to people [86]. Interestingly, in this study the MTD of PEG-rhTNF- $\alpha$

Table 6: Studies of systemic TNF- $\alpha$ with radiation +/- chemotherapy

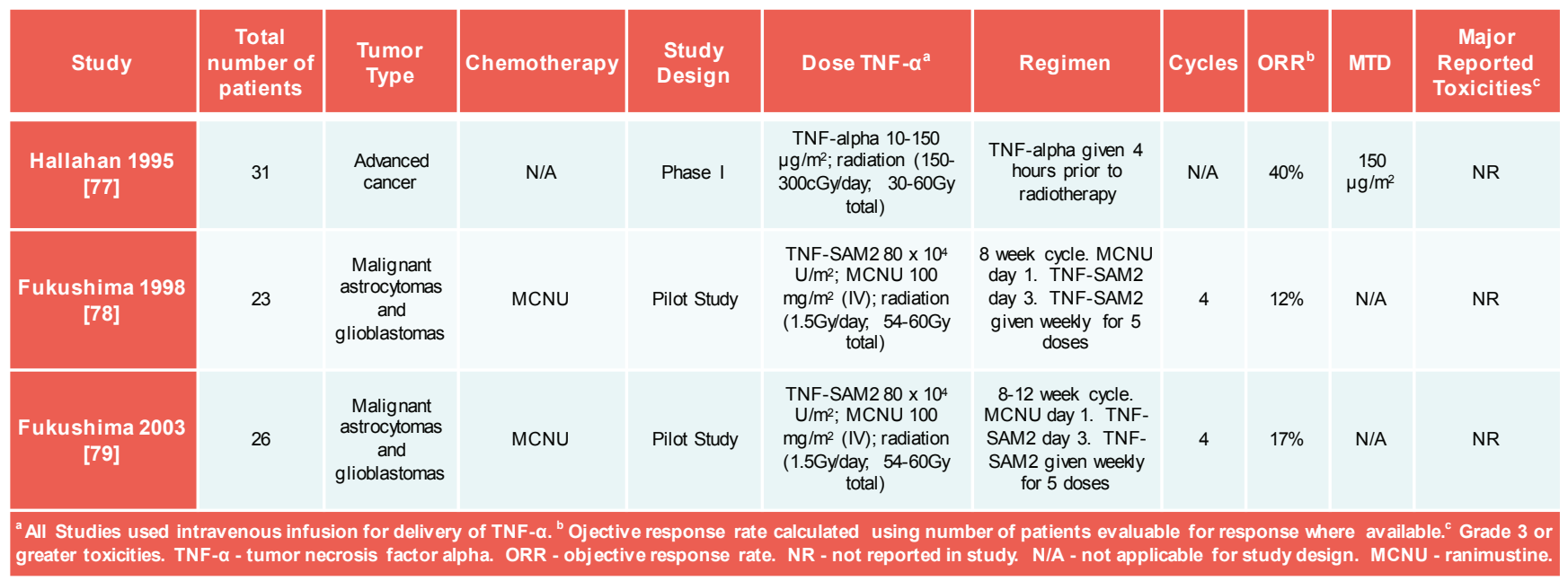


was found to be $26.7 \mu \mathrm{g} / \mathrm{kg}$ (approximately $815 \mu \mathrm{g} / \mathrm{m}^{2}$ ) and 4 of 15 dogs treated had a partial tumor response. DLT was similar to that observed with unconjugated TNF- $\alpha$, with hypotension and coagulopathy being observed. This study suggests that PEG-rhTNF- $\alpha$ may limit some of the undesirable toxicity seen with unconjugated TNF- $\alpha$ and allow for greater antitumor responses.

Asparagine-glycine-arginine conjugated to the N-terminus of TNF- $\alpha$ (NGR-TNF- $\alpha$ ) specifically binds the aminopeptidase N (CD13) of tumor vasculature [87]. CD13 is required for the pathological development of vasculature in the disease and presents an ideal target to modulate the effect of chemotherapeutics [88, 89]. Preclinical studies of NGR-TNF- $\alpha$ showed synergism with doxorubicin, cisplatin, placitaxel and gemcitabine, increasing tumor penetration of cytotoxic compounds, anticancer efficacy and decreasing treatment associated toxicity [90]. Interestingly, increase in efficacy was seen in vivo but not in vitro with tumor cell lines, indicating that this synergism is due to an indirect effect of NGRTNF- $\alpha$ on host vasculature [90]. A recent Phase Ib study of low-dose NGR-TNF- $\alpha$ with doxorubicin in advanced solid tumors demonstrated that this combination is well tolerated with no DLT observed [67]. A phase II dose of $0.8 \mu \mathrm{g} / \mathrm{m}^{2}$ of NGR-TNF- $\alpha$ and $75 \mathrm{mg} / \mathrm{m}^{2}$ of doxorubicin was recommended. The study provided hope for future development of TNF- $\alpha$ and doxorubicin combination therapy with 1 of 15 patients achieving a partial response and 10 of 15 patients with stable disease for a median duration of nearly 6 months.

An alternative concept for the use of TNF- $\alpha$ in the treatment of human cancers exists. Preclinical in vivo studies demonstrated that the uptake of radiolabeled liposomes in tumors was increased by approximately 6 fold in mice that were concomitantly treated with TNF- $\alpha$ [4]. The mechanism behind this enrichment is thought to be mediated through effects on the tumor vasculature and an enhanced-enhanced permeability and retention ( $\left.E^{2} \mathrm{PR}\right)$ effect. In vivo experiments using the combination of TNF- $\alpha$ and liposomal doxorubicin showed a significantly increased survival benefit in tumor-bearing mice treated with the combination in comparison to mice treated with either TNF- $\alpha$ or liposomal doxorubicin alone. Although single-agent liposomal doxorubicin alone delayed tumor growth and led to improved survival, the tumors eventually grew back, whereas the combination treatment with TNF- $\alpha$ and liposomal doxorubicin led to a long-term survival in $80 \%$ of the treated animals. These findings are in accordance with previously published data showing improved treatment outcomes in rat osteosarcoma and murine melanoma tumor models that were treated with lowdose TNF- $\alpha$ plus liposomal doxorubicin in comparison to TNF- $\alpha$ plus free doxorubicin $[5,91]$. The development of low-dose TNF- $\alpha$ and liposomal doxorubicin may provide unique synergy to increase efficacy and decrease toxicity of combination therapy. Clinical studies are necessary to establish the safety and efficacy of this approach. These studies are worthwhile considering the novel mechanism of synergism between TNF- $\alpha$ and liposomal doxorubicin.

\section{CONCLUSION}

TNF- $\alpha$ has been proven an effective anticancer agent in in vitro and in vivo preclinical studies. Sadly, the promise of systemic TNF- $\alpha$ has, as of yet, not translated to a patient therapy and enthusiasm has been curbed due to the toxicity profile and lack of efficacy at MTD. Combination with chemotherapy in the setting of hyperthermic isolated limb perfusion has proven quite successful, based not only on a direct anti-proliferative effect of TNF- $\alpha$, but also due to its ability to increase drug penetration into tumor tissue. The future development of systemic TNF- $\alpha$ as an anticancer treatment will rely on exploring ways to reduced systemic toxicity and exploit novel mechanisms of action to deliver greater efficacy simultaneously with decreased toxicity. A number of avenues are currently being explored based on promising preclinical and early clinical data. The novel concept of using systemic TNF- $\alpha$ to facilitate increased tumor penetration of liposomal chemotherapy seems particularly promising and worth exploring clinically.

\section{CONFLICTS OF INTEREST}

The authors declare no conflicts of interest.

\section{ACKNOWLEDGEMENTS}

This work was supported by The Virginia and D.K. Ludwig Fund for Cancer Research and the US National Institutes of Health grants CA062924 (S.Z.) and CA129825 (L.A.D.) and Swim Across America.

\section{REFERENCES}

1. Carswell EA, Old LJ, Kassel RL, Green S, Fiore N, Williamson B. An endotoxin-induced serum factor that causes necrosis of tumors. Proc Natl Acad Sci U S A. 1975; 72:3666-3670.

2. Pennica D, Nedwin GE, Hayflick JS, Seeburg PH, Derynck R, Palladino MA, Kohr WJ, Aggarwal BB, Goeddel DV. Human tumour necrosis factor: precursor structure, expression and homology to lymphotoxin. Nature. 1984; 312:724-729.

3. Jones AL, O'Brien ME, Lorentzos A, Viner C, Hanrahan A, Moore J, Millar JL, Gore ME. A randomised phase II study of carmustine alone or in combination with tumour necrosis factor in patients with advanced melanoma. Cancer Chemother Pharmacol. 1992; 30:73-76.

4. Qiao Y, Huang X, Nimmagadda S, Bai R, Staedtke V, Foss CA, Cheong I, Holdhoff M, Kato Y, Pomper MG, Riggins GJ, Kinzler KW, Diaz LA, Jr., Vogelstein B, Zhou S. A 
robust approach to enhance tumor-selective accumulation of nanoparticles. Oncotarget. 2011; 2:59-68.

5. Seynhaeve AL, Hoving S, Schipper D, Vermeulen CE, de Wiel-Ambagtsheer G, van Tiel ST, Eggermont AM, Ten Hagen TL. Tumor necrosis factor alpha mediates homogeneous distribution of liposomes in murine melanoma that contributes to a better tumor response. Cancer Res. 2007; 67:9455-9462.

6. Brouckaert P, Takahashi N, van Tiel ST, Hostens J, Eggermont AM, Seynhaeve AL, Fiers W, ten Hagen TL. Tumor necrosis factor-alpha augmented tumor response in B16BL6 melanoma-bearing mice treated with stealth liposomal doxorubicin (Doxil) correlates with altered Doxil pharmacokinetics. Int J Cancer. 2004; 109:442-448.

7. Horiuchi $\mathrm{T}$, Mitoma $\mathrm{H}$, Harashima S, Tsukamoto $\mathrm{H}$, Shimoda T. Transmembrane TNF-alpha: structure, function and interaction with anti-TNF agents. Rheumatology (Oxford). 49:1215-1228.

8. van Horssen R, Ten Hagen TL, Eggermont AM. TNF-alpha in cancer treatment: molecular insights, antitumor effects, and clinical utility. Oncologist. 2006; 11:397-408.

9. Locksley RM, Killeen N, Lenardo MJ. The TNF and TNF receptor superfamilies: integrating mammalian biology. Cell. 2001; 104:487-501.

10. Balkwill F. Tumour necrosis factor and cancer. Nat Rev Cancer. 2009; 9:361-371.

11. Parameswaran N, Patial S. Tumor necrosis factor-alpha signaling in macrophages. Crit Rev Eukaryot Gene Expr. 20:87-103.

12. Rushworth SA, Zaitseva L, Langa S, Bowles KM, MacEwan DJ. FLIP regulation of HO-1 and TNF signalling in human acute myeloid leukemia provides a unique secondary antiapoptotic mechanism. Oncotarget. 2010; 1:359-366.

13. Shirley S, Micheau O. The heme oxygenase-1 and c-FLIP in acute myeloid leukemias: two non-redundant but mutually exclusive cellular safeguards protecting cells against TNFinduced cell death? Oncotarget. 2010; 1:317-319.

14. Aggarwal BB. Signalling pathways of the TNF superfamily: a double-edged sword. Nat Rev Immunol. 2003; 3:745-756.

15. Dealtry GB, Naylor MS, Fiers W, Balkwill FR. The effect of recombinant human tumour necrosis factor on growth and macromolecular synthesis of human epithelial cells. Exp Cell Res. 1987; 170:428-438.

16. Motoo Y, Hill NO, Mahmoudi M, Osther K. Antitumor effect of human necrosis factor on human hepatoma cells PLC/PRF/5. Jpn J Exp Med. 1986; 56:151-154.

17. Sugarman BJ, Aggarwal BB, Hass PE, Figari IS, Palladino MA, Jr., Shepard HM. Recombinant human tumor necrosis factor-alpha: effects on proliferation of normal and transformed cells in vitro. Science. 1985; 230:943-945.

18. Salmon SE, Young L, Scuderi P, Clark B. Antineoplastic effects of tumor necrosis factor alone and in combination with gamma-interferon on tumor biopsies in clonogenic assay. J Clin Oncol. 1987; 5:1816-1821.
19. Asher A, Mule JJ, Reichert CM, Shiloni E, Rosenberg SA. Studies on the anti-tumor efficacy of systemically administered recombinant tumor necrosis factor against several murine tumors in vivo. J Immunol. 1987; 138:963974.

20. Haranaka K, Carswell EA, Williamson BD, Prendergast JS, Satomi N, Old LJ. Purification, characterization, and antitumor activity of nonrecombinant mouse tumor necrosis factor. Proc Natl Acad Sci U S A. 1986; 83:3949-3953.

21. Haranaka K, Satomi N, Sakurai A. Antitumor activity of murine tumor necrosis factor (TNF) against transplanted murine tumors and heterotransplanted human tumors in nude mice. Int J Cancer. 1984; 34:263-267.

22. Alexander RB, Isaacs JT, Coffey DS. Tumor necrosis factor enhances the in vitro and in vivo efficacy of chemotherapeutic drugs targeted at DNA topoisomerase II in the treatment of murine bladder cancer. J Urol. 1987; 138:427-429.

23. Krosnick JA, Mule JJ, McIntosh JK, Rosenberg SA. Augmentation of antitumor efficacy by the combination of recombinant tumor necrosis factor and chemotherapeutic agents in vivo. Cancer Res. 1989; 49:3729-3733.

24. Demidenko ZN, Blagosklonny MV. Flavopiridol induces p53 via initial inhibition of Mdm2 and p21 and, independently of p53, sensitizes apoptosis-reluctant cells to tumor necrosis factor. Cancer Res. 2004; 64:3653-3660.

25. Kikuchi A, Holan V, Minowada J. Effects of tumor necrosis factor alpha, interferon alpha and interferon gamma on non-lymphoid leukemia cell lines: growth inhibition, differentiation induction and drug sensitivity modulation. Cancer Immunol Immunother. 1992; 35:257-263.

26. Aggarwal BB, Eessalu TE, Hass PE. Characterization of receptors for human tumour necrosis factor and their regulation by gamma-interferon. Nature. 1985; 318:665667.

27. Ruggiero V, Tavernier J, Fiers W, Baglioni C. Induction of the synthesis of tumor necrosis factor receptors by interferon-gamma. J Immunol. 1986; 136:2445-2450.

28. Brouckaert PG, Leroux-Roels GG, Guisez Y, Tavernier J, Fiers W. In vivo anti-tumour activity of recombinant human and murine TNF, alone and in combination with murine IFN-gamma, on a syngeneic murine melanoma. Int J Cancer. 1986; 38:763-769.

29. Matsubara N, Fuchimoto S, Orita K. Antiproliferative effects of natural human tumor necrosis factor-alpha, interferon-alpha, and interferon-gamma on human pancreatic carcinoma cell lines. Int J Pancreatol. 1991; 8:235-243.

30. Orita K, Ando S, Kurimoto M. Synergism between human tumor necrosis factor and human interferon-alpha: effects on cells in culture. Acta Med Okayama. 1987; 41:155-160.

31. Naomoto Y, Tanaka N, Orita K. Antitumor effect of natural human tumor necrosis factor-alpha and natural human interferon-alpha in combination against human cancer 
transplanted into nude mice. Acta Med Okayama. 1989; 43:211-221.

32. Nishimura T, Ohta S, Sato N, Togashi Y, Goto M, Hashimoto Y. Combination tumor-immunotherapy with recombinant tumor necrosis factor and recombinant interleukin 2 in mice. Int J Cancer. 1987; 40:255-261.

33. Winkelhake JL, Stampfl S, Zimmerman RJ. Synergistic effects of combination therapy with human recombinant interleukin-2 and tumor necrosis factor in murine tumor models. Cancer Res. 1987; 47:3948-3953.

34. Hallahan DE, Beckett MA, Kufe D, Weichselbaum RR. The interaction between recombinant human tumor necrosis factor and radiation in 13 human tumor cell lines. Int J Radiat Oncol Biol Phys. 1990; 19:69-74.

35. Chapman PB, Lester TJ, Casper ES, Gabrilove JL, Wong GY, Kempin SJ, Gold PJ, Welt S, Warren RS, Starnes HF, et al. Clinical pharmacology of recombinant human tumor necrosis factor in patients with advanced cancer. J Clin Oncol. 1987; 5:1942-1951.

36. Creaven PJ, Plager JE, Dupere S, Huben RP, Takita H, Mittelman A, Proefrock A. Phase I clinical trial of recombinant human tumor necrosis factor. Cancer Chemother Pharmacol. 1987; 20:137-144.

37. Kimura K, Taguchi T, Urushizaki I, Ohno R, Abe O, Furue $\mathrm{H}$, Hattori T, Ichihashi H, Inoguchi K, Majima H, et al. Phase I study of recombinant human tumor necrosis factor. Cancer Chemother Pharmacol. 1987; 20:223-229.

38. Creagan ET, Kovach JS, Moertel CG, Frytak S, Kvols LK. A phase I clinical trial of recombinant human tumor necrosis factor. Cancer. 1988; 62:2467-2471.

39. Feinberg B, Kurzrock R, Talpaz M, Blick M, Saks S, Gutterman JU. A phase I trial of intravenously-administered recombinant tumor necrosis factor-alpha in cancer patients. J Clin Oncol. 1988; 6:1328-1334.

40. Sherman ML, Spriggs DR, Arthur KA, Imamura K, Frei E, 3rd, Kufe DW. Recombinant human tumor necrosis factor administered as a five-day continuous infusion in cancer patients: phase I toxicity and effects on lipid metabolism. J Clin Oncol. 1988; 6:344-350.

41. Spriggs DR, Sherman ML, Michie H, Arthur KA, Imamura K, Wilmore D, Frei E, 3rd, Kufe DW. Recombinant human tumor necrosis factor administered as a 24-hour intravenous infusion. A phase I and pharmacologic study. J Natl Cancer Inst. 1988; 80:1039-1044.

42. Taguchi T. Phase I study of recombinant human tumor necrosis factor (rHu-TNF:PT-050). Cancer Detect Prev. 1988; 12:561-572.

43. Creaven PJ, Brenner DE, Cowens JW, Huben RP, Wolf RM, Takita H, Arbuck SG, Razack MS, Proefrock AD. A phase I clinical trial of recombinant human tumor necrosis factor given daily for five days. Cancer Chemother Pharmacol. 1989; 23:186-191.

44. Jakubowski AA, Casper ES, Gabrilove JL, Templeton MA, Sherwin SA, Oettgen HF. Phase I trial of intramuscularly administered tumor necrosis factor in patients with advanced cancer. J Clin Oncol. 1989; 7:298-303.

45. Wiedenmann B, Reichardt P, Rath U, Theilmann L, Schule B, Ho AD, Schlick E, Kempeni J, Hunstein W, Kommerell B. Phase-I trial of intravenous continuous infusion of tumor necrosis factor in advanced metastatic carcinomas. J Cancer Res Clin Oncol. 1989; 115:189-192.

46. Gamm H, Lindemann A, Mertelsmann R, Herrmann F. Phase I trial of recombinant human tumour necrosis factor alpha in patients with advanced malignancy. Eur J Cancer. 1991; 27:856-863.

47. Krigel RL, Padavic-Shaller KA, Rudolph AA, Young JD, Weiner LM, Konrad M, Comis RL. Hemorrhagic gastritis as a new dose-limiting toxicity of recombinant tumor necrosis factor. J Natl Cancer Inst. 1991; 83:129-131.

48. Logan TF, Kaplan SS, Bryant JL, Ernstoff MS, Krause JR, Kirkwood JM. Granulocytopenia in cancer patients treated in a phase I trial with recombinant human tumor necrosis factor. J Immunother (1991). 1991; 10:84-95.

49. Schiller JH, Storer BE, Witt PL, Alberti D, Tombes MB, Arzoomanian R, Proctor RA, McCarthy D, Brown $\mathrm{RR}$, Voss SD, et al. Biological and clinical effects of intravenous tumor necrosis factor-alpha administered three times weekly. Cancer Res. 1991; 51:1651-1658.

50. Mittelman A, Puccio C, Gafney E, Coombe N, Singh B, Wood D, Nadler P, Ahmed T, Arlin Z. A phase I pharmacokinetic study of recombinant human tumor necrosis factor administered by a 5-day continuous infusion. Invest New Drugs. 1992; 10:183-190.

51. Furman WL, Strother D, McClain K, Bell B, Leventhal B, Pratt CB. Phase I clinical trial of recombinant human tumor necrosis factor in children with refractory solid tumors: a Pediatric Oncology Group study. J Clin Oncol. 1993; 11:2205-2210.

52. Braczkowski R, Zubelewicz B, Romanowski W, Lissoni P, Brivio F. Possible biological indicators of the response to recombinant tumour necrosis factor alpha in patients with advanced neoplasms. J Exp Clin Cancer Res. 1998; 17:349354.

53. Lenk H, Tanneberger S, Muller U, Ebert J, Shiga T. Phase II clinical trial of high-dose recombinant human tumor necrosis factor. Cancer Chemother Pharmacol. 1989; 24:391-392.

54. Heim ME, Siegmund R, Illiger HJ, Klee M, Rieche K, Berdel WE, Edler L. Tumor necrosis factor in advanced colorectal cancer: a phase II study. A trial of the phase I/II study group of the Association for Medical Oncology of the German Cancer Society. Onkologie. 1990; 13:444-447.

55. Kemeny N, Childs B, Larchian W, Rosado K, Kelsen D. A phase II trial of recombinant tumor necrosis factor in patients with advanced colorectal carcinoma. Cancer. 1990; 66:659-663.

56. Whitehead RP, Fleming T, Macdonald JS, Goodman PJ, Neefe J, Braun TJ, Swinnen LJ, Hersh EM. A phase 
II trial of recombinant tumor necrosis factor in patients with metastatic colorectal adenocarcinoma: a Southwest Oncology Group study. J Biol Response Mod. 1990; 9:588591.

57. Brown TD, Goodman P, Fleming T, Macdonald JS, Hersh EM, Braun TJ. A phase II trial of recombinant tumor necrosis factor in patients with adenocarcinoma of the pancreas: a Southwest Oncology Group study. J Immunother (1991). 1991; 10:376-378.

58. Budd GT, Green S, Baker LH, Hersh EP, Weick JK, Osborne CK. A Southwest Oncology Group phase II Trial of recombinant tumor necrosis factor in metastatic breast cancer. Cancer. 1991; 68:1694-1695.

59. Hersh EM, Metch BS, Muggia FM, Brown TD, Whitehead RP, Budd GT, Rinehart JJ, Crawford ED, Bonnet JD, Behrens BC. Phase II studies of recombinant human tumor necrosis factor alpha in patients with malignant disease: a summary of the Southwest Oncology Group experience. J Immunother (1991). 1991; 10:426-431.

60. Feldman ER, Creagan ET, Schaid DJ, Ahmann DL. Phase II trial of recombinant tumor necrosis factor in disseminated malignant melanoma. Am J Clin Oncol. 1992; 15:256-259.

61. Skillings J, Wierzbicki R, Eisenhauer E, Venner P, Letendre F, Stewart D, Weinerman B. A phase II study of recombinant tumor necrosis factor in renal cell carcinoma: a study of the National Cancer Institute of Canada Clinical Trials Group. J Immunother (1991). 1992; 11:67-70.

62. Muc-Wierzgon M, Baranowski M, Madej K. Tumor necrosis factor in advanced gastrointestinal neoplasms. A clinical trial with a focus on haematological effects. Haematologia (Budap). 1996; 27:85-92.

63. Seibel NL, Dinndorf PA, Bauer M, Sondel PM, Hammond GD, Reaman GH. Phase I study of tumor necrosis factoralpha and actinomycin $\mathrm{D}$ in pediatric patients with cancer: a Children's Cancer Group study. J Immunother Emphasis Tumor Immunol. 1994; 16:125-131.

64. Sella A, Aggarwal BB, Kilbourn RG, Bui CA, Zukiwski AA, Logothetis CJ. Phase I study of tumor necrosis factor plus actinomycin D in patients with androgen-independent prostate cancer. Cancer Biother. 1995; 10:225-235.

65. Yamamoto M, Oshiro S, Tsugu H, Hirakawa K, Ikeda K, Soma G, Fukushima T. Treatment of recurrent malignant supratentorial astrocytomas with carboplatin and etoposide combined with recombinant mutant human tumor necrosis factor-alpha. Anticancer Res. 2002; 22:2447-2453.

66. Meany HJ, Seibel NL, Sun J, Finklestein JZ, Sato J, Kelleher J, Sondel P, Reaman G. Phase 2 trial of recombinant tumor necrosis factor-alpha in combination with dactinomycin in children with recurrent Wilms tumor. J Immunother. 2008; 31:679-683.

67. Gregorc V, Santoro A, Bennicelli E, Punt CJ, Citterio G, Timmer-Bonte JN, Caligaris Cappio F, Lambiase A, Bordignon C, van Herpen CM. Phase Ib study of NGRhTNF, a selective vascular targeting agent, administered at low doses in combination with doxorubicin to patients with advanced solid tumours. Br J Cancer. 2009; 101:219-224.

68. Demetri GD, Spriggs DR, Sherman ML, Arthur KA, Imamura K, Kufe DW. A phase I trial of recombinant human tumor necrosis factor and interferon-gamma: effects of combination cytokine administration in vivo. J Clin Oncol. 1989; 7:1545-1553.

69. Kurzrock R, Feinberg B, Talpaz M, Saks S, Gutterman JU. Phase I study of a combination of recombinant tumor necrosis factor-alpha and recombinant interferon-gamma in cancer patients. J Interferon Res. 1989; 9:435-444.

70. Fiedler W, Zeller W, Peimann CJ, Weh HJ, Hossfeld DK. A phase II combination trial with recombinant human tumor necrosis factor and gamma interferon in patients with colorectal cancer. Klin Wochenschr. 1991; 69:261-268.

71. Smith JW, 2nd, Urba WJ, Clark JW, Longo DL, Farrell M, Creekmore SP, Conlon KC, Jaffe H, Steis RG. Phase I evaluation of recombinant tumor necrosis factor given in combination with recombinant interferon-gamma. J Immunother (1991). 1991; 10:355-362.

72. Schiller JH, Witt PL, Storer B, Alberti D, Tombes MB, Arzoomanian R, Brown RR, Proctor RA, Voss SD, Spriggs $\mathrm{DR}$, et al. Clinical and biologic effects of combination therapy with gamma-interferon and tumor necrosis factor. Cancer. 1992; 69:562-571.

73. Yang SC, Grimm EA, Parkinson DR, Carinhas J, Fry KD, Mendiguren-Rodriguez A, Licciardello J, Owen-Schaub LB, Hong WK, Roth JA. Clinical and immunomodulatory effects of combination immunotherapy with low-dose interleukin 2 and tumor necrosis factor alpha in patients with advanced non-small cell lung cancer: a phase I trial. Cancer Res. 1991; 51:3669-3676.

74. Krigel RL, Padavic-Shaller K, Toomey C, Comis RL, Weiner LM. Phase I study of sequentially administered recombinant tumor necrosis factor and recombinant interleukin-2. J Immunother Emphasis Tumor Immunol. 1995; 17:161-170.

75. Schiller JH, Morgan-Ihrig C, Levitt ML. Concomitant administration of interleukin-2 plus tumor necrosis factor in advanced non-small cell lung cancer. Am J Clin Oncol. $1995 ; 18: 47-51$.

76. Eskander ED, Harvey HA, Givant E, Lipton A. Phase I study combining tumor necrosis factor with interferonalpha and interleukin-2. Am J Clin Oncol. 1997; 20:511514.

77. Hallahan DE, Vokes EE, Rubin SJ, O'Brien S, Samuels B, Vijaykumar S, Kufe DW, Phillips R, Weichselbaum RR. Phase I dose-escalation study of tumor necrosis factoralpha and concomitant radiation therapy. Cancer J Sci Am. 1995; 1:204-209.

78. Fukushima T, Yamamoto M, Ikeda K, Tsugu H, Kimura $\mathrm{H}$, Soma G, Tomonaga M. Treatment of malignant astrocytomas with recombinant mutant human tumor necrosis factor-alpha (TNF-SAM2). Anticancer Res. 1998; 
18:3965-3970.

79. Fukushima T, Yamamoto $\mathrm{M}$, Oshiro $\mathrm{S}$, Tsugu $\mathrm{H}$, Hirakawa K, Soma G. Recombinant mutant human tumor necrosis factor-alpha (TNF-SAM2) immunotherapy with ranimustine chemotherapy and concurrent radiation therapy for malignant astrocytomas. Anticancer Res. 2003; 23:4473-4481.

80. Harris JM, Chess RB. Effect of pegylation on pharmaceuticals. Nat Rev Drug Discov. 2003; 2:214-221.

81. Tsutsumi Y, Kihira T, Tsunoda S, Kubo K, Miyake M, Kanamori T, Nakagawa S, Mayumi T. Intravenous administration of polyethylene glycol-modified tumor necrosis factor-alpha completely regressed solid tumor in Meth-A murine sarcoma model. Jpn J Cancer Res. 1994; 85:1185-1188.

82. Tsutsumi Y, Kihira T, Yamamoto S, Kubo K, Nakagawa S, Miyake M, Horisawa Y, Kanamori T, Ikegami H, Mayumi T. Chemical modification of natural human tumor necrosis factor-alpha with polyethylene glycol increases its antitumor potency. Jpn J Cancer Res. 1994; 85:9-12.

83. Li YP, Pei YY, Ding J, Shen ZM, Zhang XY, Gu ZH, Zhou JJ. PEGylated recombinant human tumor necrosis factor alpha: preparation and anti-tumor potency. Acta Pharmacol Sin. 2001; 22:549-555.

84. Tsutsumi Y, Kihira T, Tsunoda S, Kamada H, Nakagawa S, Kaneda Y, Kanamori T, Mayumi T. Molecular design of hybrid tumor necrosis factor-alpha III: polyethylene glycol-modified tumor necrosis factor-alpha has markedly enhanced antitumor potency due to longer plasma half-life and higher tumor accumulation. J Pharmacol Exp Ther. 1996; 278:1006-1011.

85. Thamm DH, Kurzman ID, Clark MA, Ehrhart EJ, 3rd, Kraft SL, Gustafson DL, Vail DM. Preclinical investigation of PEGylated tumor necrosis factor alpha in dogs with spontaneous tumors: phase I evaluation. Clin Cancer Res. 16:1498-1508.

86. Paoloni M, Khanna C. Translation of new cancer treatments from pet dogs to humans. Nat Rev Cancer. 2008; 8:147156.

87. Curnis F, Sacchi A, Borgna L, Magni F, Gasparri A, Corti A. Enhancement of tumor necrosis factor alpha antitumor immunotherapeutic properties by targeted delivery to aminopeptidase N (CD13). Nat Biotechnol. 2000; 18:11851190.

88. Corti A, Curnis F, Arap W, Pasqualini R. The neovasculature homing motif NGR: more than meets the eye. Blood. 2008; 112:2628-2635.

89. Curnis F, Sacchi A, Corti A. Improving chemotherapeutic drug penetration in tumors by vascular targeting and barrier alteration. J Clin Invest. 2002; 110:475-482.

90. Sacchi A, Gasparri A, Gallo-Stampino C, Toma S, Curnis F, Corti A. Synergistic antitumor activity of cisplatin, paclitaxel, and gemcitabine with tumor vasculaturetargeted tumor necrosis factor-alpha. Clin Cancer Res.
2006; 12:175-182.

91. Hoving S, Seynhaeve AL, van Tiel ST, Eggermont AM, ten Hagen TL. Addition of low-dose tumor necrosis factoralpha to systemic treatment with STEALTH liposomal doxorubicin (Doxil) improved anti-tumor activity in osteosarcoma-bearing rats. Anticancer Drugs. 2005; 16:667-674. 